\title{
Chaos in wavy-stratified fluid-fluid flow
}

\author{
Cite as: Chaos 29, 033121 (2019); doi: 10.1063/1.5055782
}

Submitted: 10 September 2018 . Accepted: 20 February 2019.

Published Online: 18 March 2019

\author{
Avinash Vaidheeswaran, ${ }^{1,2}$ (i) Alejandro Clausse, ${ }^{3}$ William D. Fullmer, ${ }^{1,4}$ Raul Marino, ${ }^{5}$ and \\ Martin Lopez de Bertodano ${ }^{6, a)}$
}

\author{
AFFILIATIONS \\ ${ }^{1}$ National Energy Technology Laboratory, Morgantown, West Virginia 26507, USA \\ ${ }^{2}$ West Virginia University Research Corporation, Morgantown, West Virginia 26506, USA \\ ${ }^{3}$ CNEA-CONICET and Universidad Nacional del Centro, 7000 Tandil, Argentina \\ ${ }^{4}$ Leidos, Morgantown, West Virginia 26507, USA \\ ${ }^{5}$ Facultad de Ciencias Exactas y Naturales, Universidad Nacional de Cuyo, 5500 Mendoza, Argentina \\ ${ }^{6}$ School of Nuclear Engineering, Purdue University, West Lafayette, Indiana 47907, USA
}

a) Electronic mail: bertodan@purdue.edu

\begin{abstract}
We perform a nonlinear analysis of a fluid-fluid wavy-stratified flow using a simplified two-fluid model (TFM), i.e., the fixed-flux model (FFM), which is an adaptation of the shallow water theory for the two-layer problem. Linear analysis using the perturbation method illustrates the shortwave physics leading to the Kelvin-Helmholtz instability (KHI). The interface dynamics are chaotic, and analysis beyond the onset of instability is required to understand the nonlinear evolution of waves. The two-equation FFM solver based on a higher-order spatiotemporal finite difference scheme is used in the current simulations. The solution methodology is verified, and the results are compared with the measurements from a laboratory-scale experiment. The finite-time Lyapunov exponent (FTLE) based on simulations is comparable and slightly higher than the autocorrelation function decay rate, consistent with previous findings. Furthermore, the FTLE is observed to be a strong function of the angle of inclination, while the root mean square of the interface height exhibits a square-root dependence. It is demonstrated that this simple 1-D FFM captures the essential chaotic features of the interface dynamics. This study also adds to a growing body of work indicating that a TFM with appropriate short wavelength physics is well-behaved and chaotic beyond the KHI.
\end{abstract}

Published under license by AIP Publishing. https://doi.org/10.1063/1.5055782

\begin{abstract}
Multiphase flows-the simultaneous motion of multiple components which may be in different states of matter and are often characterized by an interface separating the components-are frequently observed in nature and industrial applications. Multiphase flows appear in a variety of geometrical configurations (flow regimes) depending on the properties of the constituents and the flow conditions. Here, we study wavy-stratified flows of two immiscible liquids having a distinct interface, flowing counter-currently in a circular pipe. At flow rates beyond a critical value, the Kelvin-Helmholtz instability leads to a nonlinear evolution of the fluid-fluid interface. We present our findings from the numerical analysis of a slightly inclined gasoline-water system and compare the results with the bench-scale experiment. We use a simplified two-fluid model-Eulerian-Eulerian model in which both phases are treated as inter-penetrating continuua-called the fixed-flux model and highlight the simple models' ability to predict the chaotic evolution of the interface.
\end{abstract}

\section{INTRODUCTION}

Applications involving fluid dynamics often exhibit nonlinear behavior. Chaos in single phase flows has been extensively studied in the past and still remains an area of active research. Transition to turbulence is a classic example where underlying strange attractors characterize the flow. ${ }^{1}$ When an additional component or phase is present, new interfacial phenomena occur. Multiphase flows are known to exhibit hydrodynamic instabilities when differences in densities or velocities between the constituents exceed a critical limit. Chaos analysis of such systems is limited and includes the works of Pence and Beasley ${ }^{2}$ and Fullmer and Hrenya. ${ }^{3}$ In this article, we analyze the Kelvin-Helmholtz instability (KHI) which gives rise to well-known features including formation of clouds, waves in oceans, and Saturn's magnetopause. ${ }^{4}$ It is also important in industrial applications such as transport of oil and gas ${ }^{5}$ and emergency core cooling systems of nuclear power reactors. 
One of the first known measurements on the fluid-fluid interface dynamics was reported by Thorpe 7 for a kerosene-water stratified flow in a rectangular channel. The experiments had a limited duration, sufficient to analyze the transition to the wavystratified flow but not adequate for the subsequent evolution of waves. Recently, Duponcheel et al. ${ }^{6}$ replicated the experiments of Thorpe and used the same geometry to obtain high-quality data. In view of extending the analysis well past the inception of KHI, Vaidheeswaran et al. ${ }^{8}$ performed experiments using gasoline and water in a round pipe. The post-processed data are presented here to further analyze the nonlinear evolution of waves. It is important to note that the conditions chosen for the experiment are such that the individual phases remain in the laminar flow regime. Hence, chaos arises from a multiphase interfacial instability and not from shear-induced turbulence.

Linear stability analysis (LSA) of the KHI is used to determine the mathematical nature of the system of equations. LSA can also help identify terms which stabilize the model and prevent immediate numerical divergence, i.e., terms that make the model well-posed. For instance, higher-order terms present in the TFM have been proven to influence the stability of the system of equations ${ }^{9-14}$ without completely hyperbolizing the model, i.e., no growth at all wavelengths. However, based on LSA of a well-posed TFM, there persists exponential growth at finite wavelengths, which is bounded by the inherent nonlinearity. ${ }^{15}$ The nonlinear interaction between material shock waves and viscosity stabilizes the system by transferring growth at long wavelengths to dissipative short wavelengths.

In this work, we use a Fixed-Flux Model (FFM), ${ }^{16}$ which is a simplified two-equation form of the TFM. An advantage of the FFM is that the pressure gradient terms are not present and hence the numerical integration is straightforward. In Sec. II, the watergasoline experiments are described followed by the description of the FFM for the stratified fluid-fluid flow in Sec. III. Stability of the hyperbolic-dispersive FFM is then discussed in the context of LSA and numerical simulation in Secs. IV A and IV B. The work is concluded with a brief summary and outlook in Sec. V.

\section{EXPERIMENT}

In this work, we compare numerical simulations and experiments conducted previously by Vaidheeswaran et al. ${ }^{8}$ and de Bertodano et al. ${ }^{16}$ Additionally, a novel analysis of the experimental data is provided herein. Thorpe ${ }^{7}$ had previously performed similar experiments in a rectangular channel using water and kerosene. More recently, Duponcheel et al. ${ }^{6}$ used an identical geometry to reproduce some of the results of the original Thrope experiment. Besides, a second pair of working fluids, water and $n$-hexane, were used to observe the effect of material properties on the KHI. Their interest was in the initial development of unstable waves. Conversely, the objective while designing the experiments used here was to further explore the chaotic nature of the fluid-fluid interface. Hence, the following modifications were made compared to the setup of Thorpe $^{7}$ and Duponcheel et al.: ${ }^{6}$ (i) increase the $L / H$ ratio $(L=$ $2.4 \mathrm{~m}, H=D=0.02 \mathrm{~m}$ ) to allow further development of waves and obtain statistics for a longer duration and (ii) reduce $D$ to ensure that the flow does not undergo transition to turbulence. The fluids used were water (heavier phase) and gasoline (lighter phase)

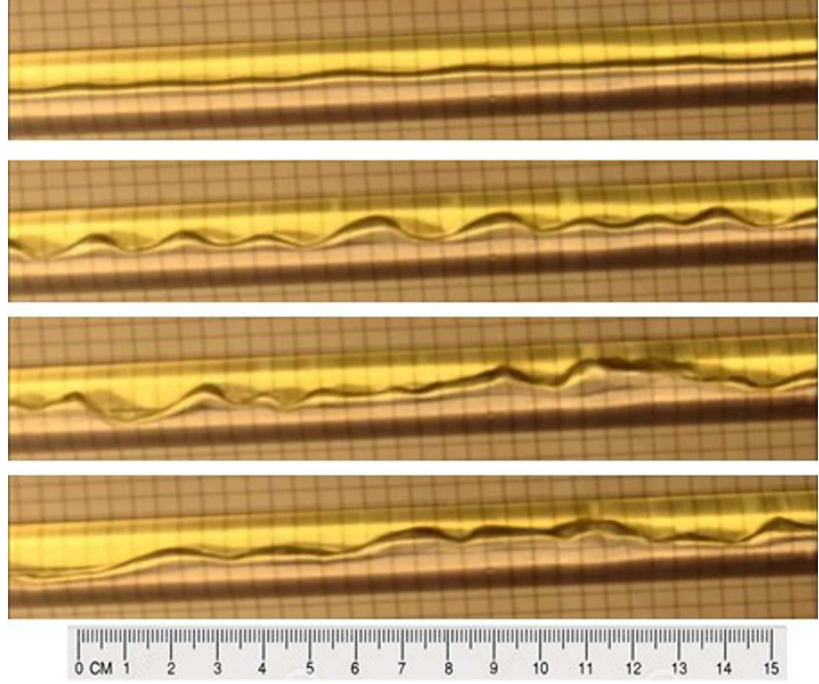

FIG. 1. Evolution of the wavy-stratified flow in water-gasoline experiments at $3.1^{\circ}$ inclination. Instantaneous snapshots correspond to (from top to bottom) $t=0 \mathrm{~s}$, $0.08 \mathrm{~s}, 0.32 \mathrm{~s}$, and $8 \mathrm{~s}$, respectively.

having the following material properties: $\rho_{1}=1000 \mathrm{~kg} / \mathrm{m}^{3}, \rho_{2}=$ $720 \mathrm{~kg} / \mathrm{m}^{3}, \mu_{1}=0.001 \mathrm{Pas}, \mu_{2}=0.0005 \mathrm{~Pa} \mathrm{~s}$, and $\sigma=0.04 \mathrm{~N} / \mathrm{m}$. Subscripts 1 and 2 refer to water and gasoline, respectively. The test section was sealed at both the ends and was initially held at rest to allow the mixture to settle. Once complete stratification occurs, the apparatus was tilted by $3.1^{\circ}$ which initiates a counter-current flow pattern. The interfacial profile was measured with a video camera. The chosen setup and conditions increase the duration of the experiments and isolate the chaotic behavior related to the interfacial $\mathrm{KHI}$ from turbulence.

Figure 1 shows the evolution of the wavy-interface which indicates initial growth phase and saturation. The initial growth is qualitatively similar to the rectangular channel experiments of Thorpe. In this work, we extend the original experimental dataset ${ }^{8,16}$ by computing the autocorrelation function (ACF) of the wavy interface from the high-speed visualization. The images were digitized and the liquid level was detected using the Canny edge detector algorithm. ${ }^{17}$ The level signals at ten selected positions along the pipe were postprocessed to generate the best estimate for the ACF.

\section{FIXED-FLUX TWO-FLUID MODEL}

The $\mathrm{TFM}^{18-20}$ provides an Eulerian description of the multiphase flow dynamics, where the constituent phases are treated as inter-penetrating continua. Based on the dimensionality of the problem, further simplifications can be made. The 1-D TFM is derived from the 3-D Eulerian TFM by averaging across the crosssectional area. The 1-D TFM may be further reduced to an FFM for wavy-stratified flows, which is a variation of the shallow water theory consisting of two coupled partial differential equations. It is worth mentioning that such an FFM could be derived for dispersed bubbly flows as well. ${ }^{21}$ As the term fixed-flux suggests, 


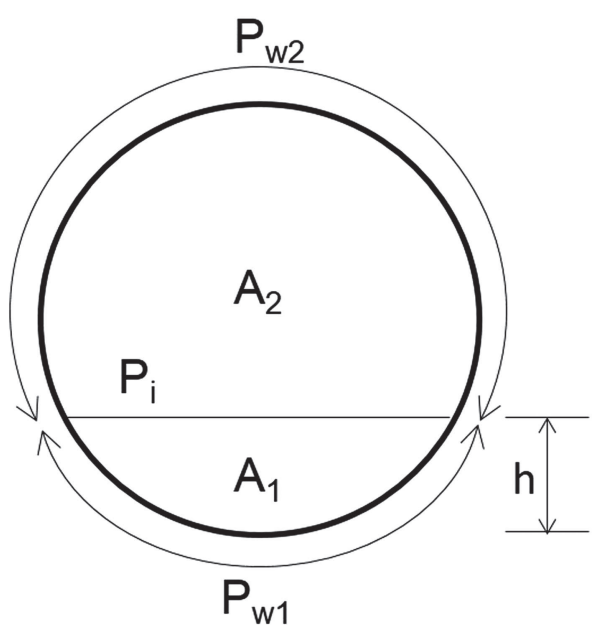

FIG. 2. Geometrical representation of a pipe cross-section (diameter $D$ ) having heavier fluid at the bottom and lighter fluid at the top.

the simplification involves assuming a constant volumetric flux, $j=\alpha_{1} u_{1}+\left(1-\alpha_{1}\right) u_{2}$. Here, we apply the 1-D FFM for the wavy-stratified pipe flow, Fig. 2, developed by Bertodano et al. ${ }^{16}$ The FFM continuity and momentum equations are given by

$$
\frac{\partial \alpha_{1}}{\partial t}+\frac{\partial}{\partial x}\left(\alpha_{1} u_{1}\right)=0
$$

and

$$
\frac{\partial u_{1}}{\partial t}+B_{21} \frac{\partial \alpha_{1}}{\partial x}+B_{22} \frac{\partial u_{1}}{\partial x}=C_{\alpha_{1}}\left(F_{\sigma}+F_{v}+F\right),
$$

respectively, where

$$
C_{\alpha_{1}}=\frac{1-\alpha_{1}}{1-\alpha_{1}+r_{\rho} \alpha_{1}} .
$$

The convective coefficients are given by

$$
\begin{aligned}
B_{21}= & -C_{\alpha_{1}}\left[\left(\frac{1}{1-\alpha_{1}}+\frac{1}{3}\right) r_{\rho}\left(u_{2}-u_{1}\right)^{2}-\frac{1}{3}\left(1+r_{\rho}\right) u_{1}^{2}\right. \\
& \left.-\frac{\pi}{4} \sin \left[\frac{\pi}{2}\left(\frac{1}{2}+\alpha_{1}\right)\right]\left(1-r_{\rho}\right) g_{y} D\right]
\end{aligned}
$$

and

$$
\begin{aligned}
B_{22}= & C_{\alpha_{1}}\left[u_{1}+r_{\rho} \frac{\alpha_{1}}{1-\alpha_{1}}\left(2 u_{2}-u_{1}\right)\right. \\
& \left.+\frac{2}{3} \frac{\alpha_{1}}{1-\alpha_{1}}\left(1-\alpha_{1}-r_{\rho} \alpha_{1}\right) u_{1}\right] .
\end{aligned}
$$

The forces on the right hand side (RHS) of Eq. (2) are due to the surface tension,

$$
F_{\sigma}=\frac{\sigma D}{2 \rho_{1}} \frac{\partial^{3}}{\partial x^{3}}\left\{1-\cos \left[\frac{\pi}{2}\left(\frac{1}{2}+\alpha_{1}\right)\right]\right\}
$$

viscous diffusion,

$$
F_{v}=v_{T}\left[\left(\frac{1}{\alpha_{1}}+\frac{r_{\rho}}{\alpha_{2}}\right) \frac{\partial}{\partial x}\left(\alpha_{1} \frac{\partial u_{1}}{\partial x}\right)+\frac{r_{\rho}}{\alpha_{2}} \frac{\partial}{\partial x}\left(\frac{u_{1}}{\alpha_{2}} \frac{\partial \alpha_{1}}{\partial x}\right)\right],
$$

and wall and interfacial drag,

$$
\begin{aligned}
F= & \left(1-r_{\rho}\right) g_{x}-16 \frac{P_{w 1} v_{1}}{\alpha_{1} A D_{h 1}} u_{1}+16 \frac{P_{w 2} v_{2}}{\alpha_{2} A D_{h 2}} r_{\rho} u_{2} \\
& +\frac{1}{\alpha_{1} \alpha_{2}} \frac{P_{i} v_{2}}{A D_{h 2}} f_{i} r_{\rho} u_{2} .
\end{aligned}
$$

The phasic hydraulic diameters are defined as

$$
D_{h i}=4 \frac{\alpha_{i} A}{P_{w i}+P_{i}} .
$$

The interfacial friction factor, $f_{i}$, is adopted from the work of Andritsos and Hanratty, 22

$$
f_{i}=1+15 \sqrt{\alpha_{1}}\left[\frac{u_{2}-u_{1}}{\left(u_{2}-u_{1}\right)_{c}}-1\right] .
$$

\section{RESULTS}

\section{A. Linear stability analysis}

The growth characteristics of different wave components are determined using LSA. Equations (1) and (2) are recast into the following vector form:

$$
\mathbf{A} \frac{\partial \underline{\phi}}{\partial t}+\mathbf{B} \frac{\partial \underline{\phi}}{\partial x}+\mathbf{D} \frac{\partial^{2} \underline{\phi}}{\partial x^{2}}+\mathbf{E} \frac{\partial^{3} \underline{\underline{\phi}}}{\partial x^{3}}=0,
$$

where $\phi=\left[\alpha_{1} u_{1}\right]^{T}$. Note that the force terms given by Eq. (8) are algebraic functions of the state variables and thus do not affect the character of the linear dynamics past the KHI. They are, nevertheless, essential for the kinematic instability that occurs before the KHI. ${ }^{16}$ Therefore, algebraic forces are neglected here for assessing the linear stability of the coupled system of equations. These terms are, however, included while performing numerical simulations. Perturbing the base state and linearizing Eq. (11) gives

$$
\operatorname{Det}\left[-i \omega \mathbf{A}+i k_{\lambda} \mathbf{B}+\left(i k_{\lambda}\right)^{2} \mathbf{D}+\left(i k_{\lambda}\right)^{3} \mathbf{E}\right]=0 .
$$

The solution to Eq. (12) is the dispersion relation between growth rate $\omega$ and wave number $k_{\lambda}$. Figure 3 shows the plots of magnitude of the imaginary component of $\omega$ as a function of wavelength $\lambda=2 \pi / k_{\lambda}$ for conditions beyond the critical KHI.

When the phasic viscosity or surface tension is neglected, the growth rate is unbounded as $\lambda \rightarrow 0$. This constitutes the ill-posed condition beyond the KHI limit. This is the same result obtained using the 2-D Euler equations for a vortex sheet. Including kinematic viscosity removes the unboundedness (ill-posedness) $;^{23}$ however, the zero wavelength growth rate, $\omega_{i}(\lambda=0)=100 \mathrm{~s}^{-1}$ remains critical (most unstable). This appears quite similar to the ill-posed model over a majority of the frequency spectrum. Numerically, such a model would behave like the ill-posed model manifesting in an extremely grid-dependent instability (ill-behaved). On the other hand, the surface tension renders the model well-posed and well-behaved with a finite valued cut-off wavelength, $\lambda_{c}$, below which the model is either 


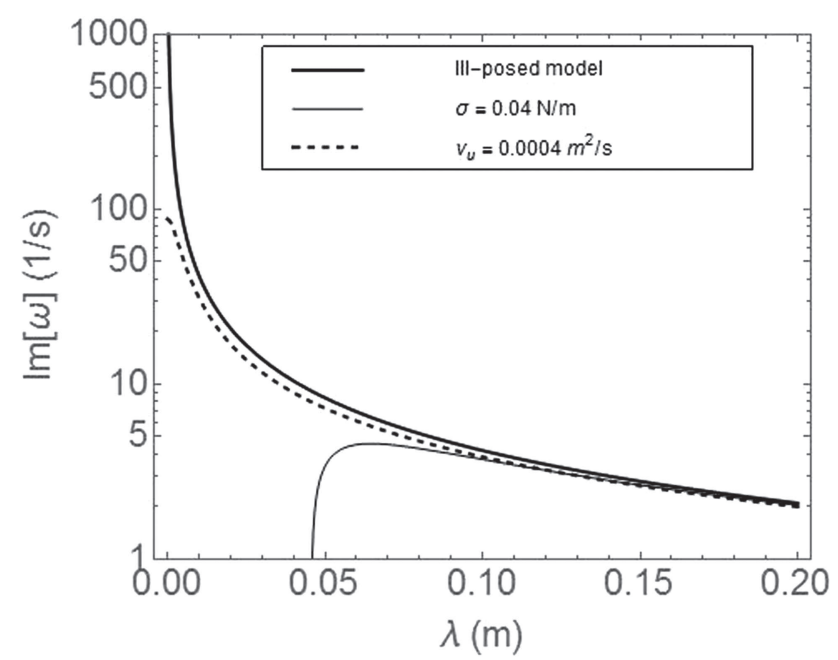

FIG. 3. Dispersion relation for the FFMs using Eq. (12). The curves correspond to the basic ill-posed model (unbounded exponential growth for $\lambda \rightarrow 0$ ), FFM with kinematic viscosity (bounded exponential growth for $\lambda \rightarrow 0$ ) and FFM with the surface tension (well-posed).

neutrally stable (inviscid) or dissipative (viscous). The cut-off wavelength is approximately $\lambda_{0}=5 \mathrm{~cm}$ for the water-gasoline system. The results obtained are consistent with the work of Ramshaw and Trapp 9 who were the first to demonstrate the well-posed behavior of 1-D TFM using surface tension. Although the model including surface tension has been rendered well-posed, linear stability still indicates that finite wavelengths will grow at an exponential rate. In Sec. IV B, we turn to nonlinear analysis of numerical simulations of this linearly unstable model. The kinematic viscosity turns out to play a key role in the Lyapunov stability of the model.

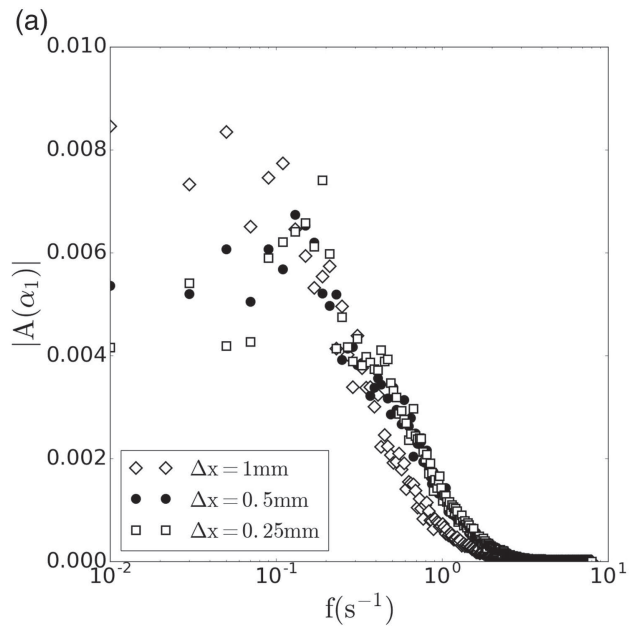

\section{B. Numerical simulations}

Equations (1) to (10) are solved using an in-house FFM solver originally developed for the prototypic Kreiss-Yström equations. ${ }^{24}$ The SMART flux-limiter scheme ${ }^{25}$ is used for spatial discretization, and time marching is done by means of a strong stability preserving three-step, third-order Runge-Kutta method. ${ }^{26}$ The chosen scheme reduces numerical diffusion while preserving numerical stability. This is essential to model the inception of material shocks and wave cusps which could get damped through excess diffusion. Hence, the use of a first-order discretization scheme or coarse grid has been avoided. Furthermore, past the onset of KHI, the nonlinear wave dynamics depend on the interaction between steep wave-fronts and viscous diffusion. It is important that the system is not over-damped through numerical artifacts.

A spectral convergence test is used to ensure numerical stability, i.e., that the discretization scheme has not resulted in an ill-behaved numerical model. Three grid levels below $\lambda_{0}$ are used: $\Delta x=1,0.5$, and $0.25 \mathrm{~mm}$. The corresponding time step sizes are $\Delta t=0.2,0.1$, and $0.05 \mathrm{~ms}$, respectively. The domain length is the same as the pipe length used for the experiments, but periodic boundary conditions are used to approximate the behavior in an infinite channel. This enabled us to perform numerical experiments over long periods of time in order to collect adequate statistics. The domain was initialized using a slightly perturbed liquid volume fraction field given by

$$
\alpha_{1}(x, 0)=\alpha_{1,0}+\delta\left[\exp \left(\frac{x-x_{01}}{\lambda_{01}}\right)^{2}-\exp \left(\frac{x-x_{02}}{\lambda_{02}}\right)^{2}\right],
$$

where $\delta=0.02, \alpha_{1,0}=0.542, \lambda_{01}=\lambda_{02}=0.02 \mathrm{~m}, x_{01}=0.45 \mathrm{~m}$, and $x_{02}=0.55 \mathrm{~m}$. The use of a relatively large amplitude disturbance was previously found ${ }^{27}$ to help moderate the transition from an initial condition to a fully-developed (unstable) state. Other perturbations also lead to same results in a statistical sense, as long as they are contained in the basin of attraction of the dynamic invariant. A constant

(b)

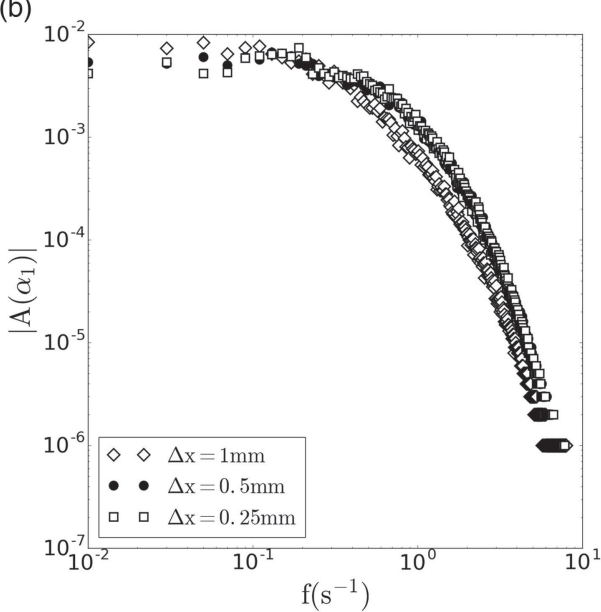

FIG. 4. Fourier spectra from $\alpha_{1}$ time series based on successive grid refinements in (a) log-linear plot and (b) log-log plot. 

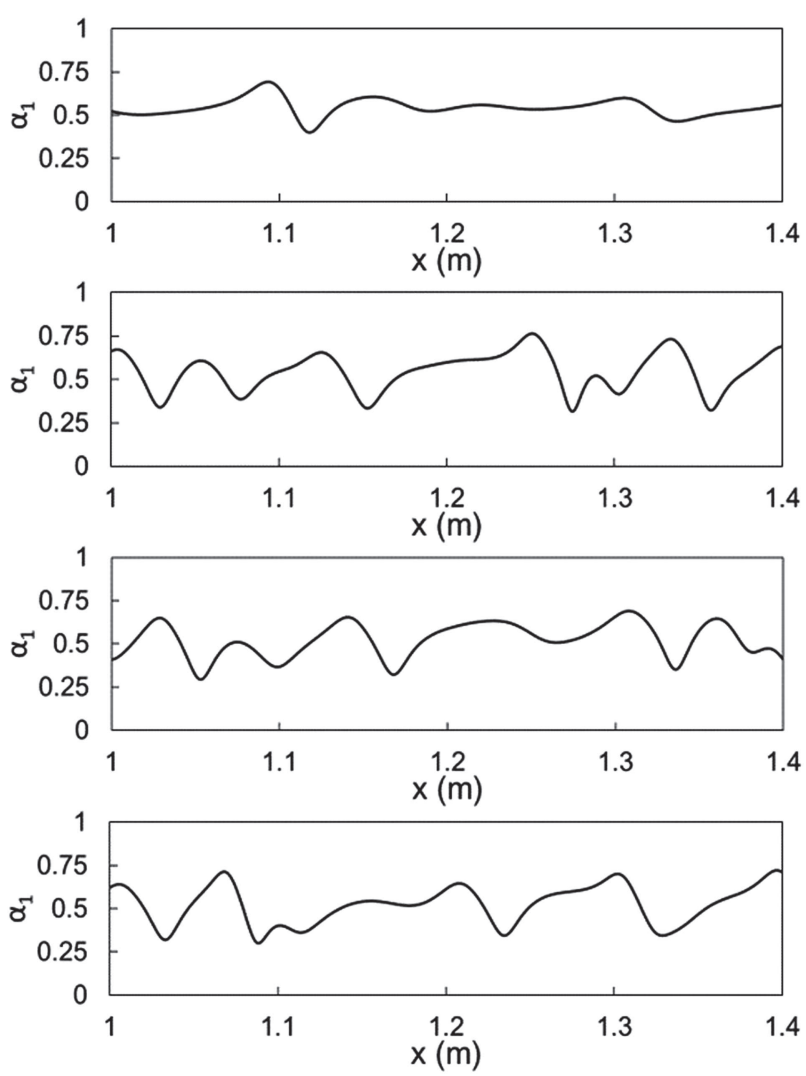
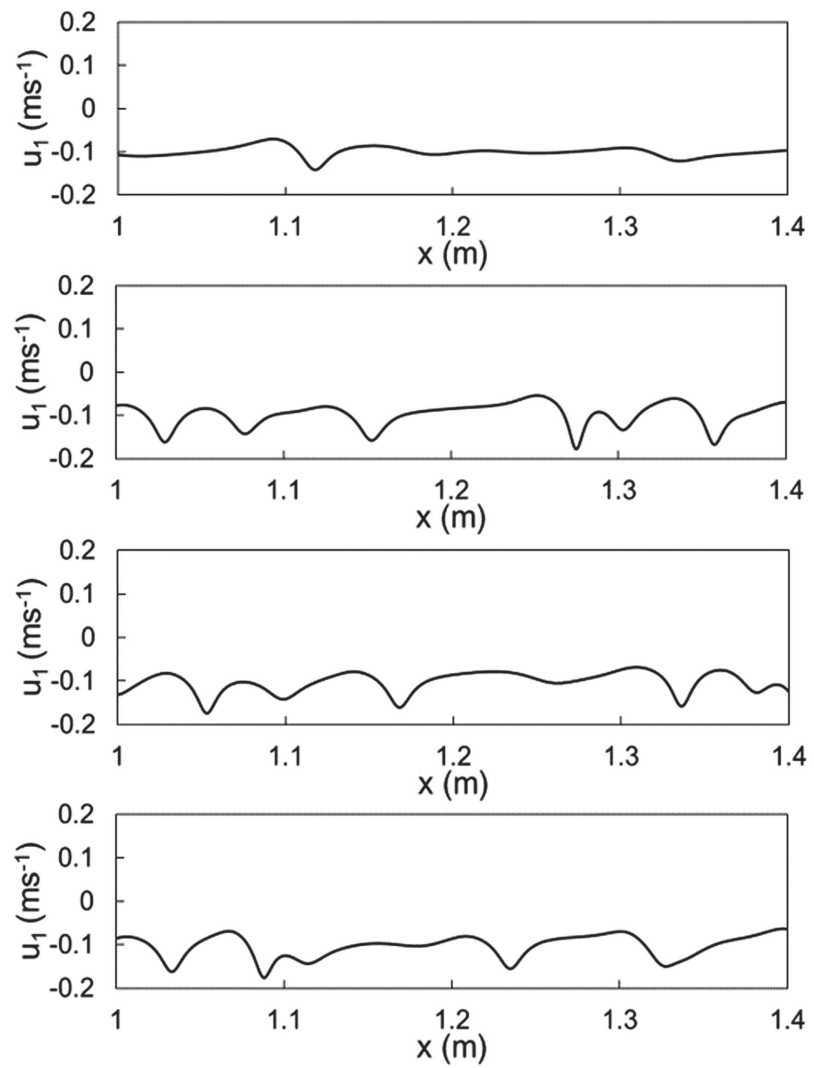

FIG. 5. Instantaneous $\alpha_{1}$ and $u_{1}$ distributions from FFM simulation at $2 \mathrm{~s}, 4 \mathrm{~s}, 8 \mathrm{~s}$, and $15 \mathrm{~s}$ (top to bottom), respectively.

velocity field is specified, $u_{1}=-0.1 \mathrm{~m} / \mathrm{s}$, calculated from the kinematic (quasi-static) condition, i.e., by solving $F=0$ in Eq. (8). The resulting FFT spectra in Fig. 4 shows that the long-wavelength (low frequency) energy generated from the linear KHI is transferred to higher frequencies through nonlinear dissipation, resulting in a continuous distribution of Fourier modes. The spectra corresponding to $\Delta x=0.5 \mathrm{~mm}$ and $\Delta x=0.25 \mathrm{~mm}$ indicate remarkable statistical convergence. Therefore, we use the more computationally affordable $\Delta x=0.5 \mathrm{~mm}$ for further analysis.

Typical waveforms produced in the simulations are shown in Fig. 5 for an inclination of $2.4^{\circ}$ along with $u_{1}$ profiles. Qualitatively, the waves are very similar to those observed in the experiments (see Fig. 1). Quantitatively, however, the dominant wavelength observed in the simulation is approximately twice as large compared to the experimental results. The discrepancy is likely due to the fact that this is not a direct ("apples-to-apples") comparison. The following are some of the notable differences between the experiment and the present simulations. First, the experiments have a finite boundary and duration while the simulations are performed using periodic boundaries. In the experiment, the channel is suddenly tilted, the flow accelerates from rest, and the channel ends fill up with single-phase fluids. Because the FFM is singular in the single-phase limit, the finite-time experiment has been compared to an essentially infinite-time, periodic geometry. Second, the onset of transition to the wavy-stratified flow is very sensitive to the constitutive relation for interfacial force. The associated uncertainties make it challenging to accurately predict the short-term behavior of the experimental system. However, our objective is to analyze the nonlinear mechanism that bounds the system well-past the inception of the KHI. Finally, the 1-D assumption is a significant simplification of the wavy-stratified flow in a pipe and does not consider multi-dimensional effects such as boundary layer development or its interactions with the interface dynamics.

The finite-time Lyapunov exponent (FTLE) is approximated using nearby initial states. ${ }^{16,24}$ For a given angle of inclination, the initial condition [Eq. (13)] is perturbed by a small number, $10^{-8}$, approximately the square root of the double precision accuracy of the digital implementation. The $\mathrm{L}_{2}$-norm of deviation in the trajectories relative to the base (unperturbed) state is calculated as a function of time and best-fit with an exponential curve to estimate the FTLE. This procedure is repeated at multiple angles of inclination. Figure 6 shows that the FTLE has a strong dependence on $\theta$ beyond the onset of KHI. With an increase in the angle of inclination, the relative velocity between the components increases making the fluid-fluid system more unstable. This occurs in conjunction with an increase in the magnitude of the FTLE. Also shown in Fig. 6 is the root mean square 


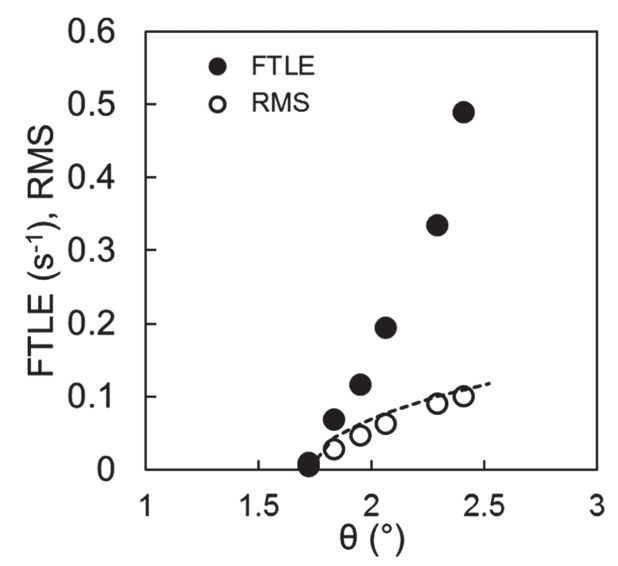

FIG. 6. Finite time Lyapunov exponent and root mean square evaluated using $\alpha_{1}$ time series. The dashed curve represents the dependence proportional to $\left(\theta-\theta_{c}\right)^{0.5}$

(RMS) of the liquid volume fraction $\sim\left(\theta-\theta_{c}\right)^{0.5}$, which is a measure of the amplitude of sustained oscillations. $\theta_{c}=1.72^{\circ}$ is the critical angle when the system becomes chaotic. The second derivative of the RMS is negative, which suggests that a supercritical type of bifurcation occurs at $\theta_{c}$. Beyond approximately $2.4^{\circ}$, the waves become so chaotic that the interface reaches the top or bottom of the channel, leading to singularity in FFM.

Beyond sensitivity to initial conditions (quantified by the positive values of FTLE), the decay of correlations is another characteristic feature of chaos. Unlike FTLE, ACF is more easily obtained from physical experiments. Here, we compare the ACF using discrete $\alpha_{1}$

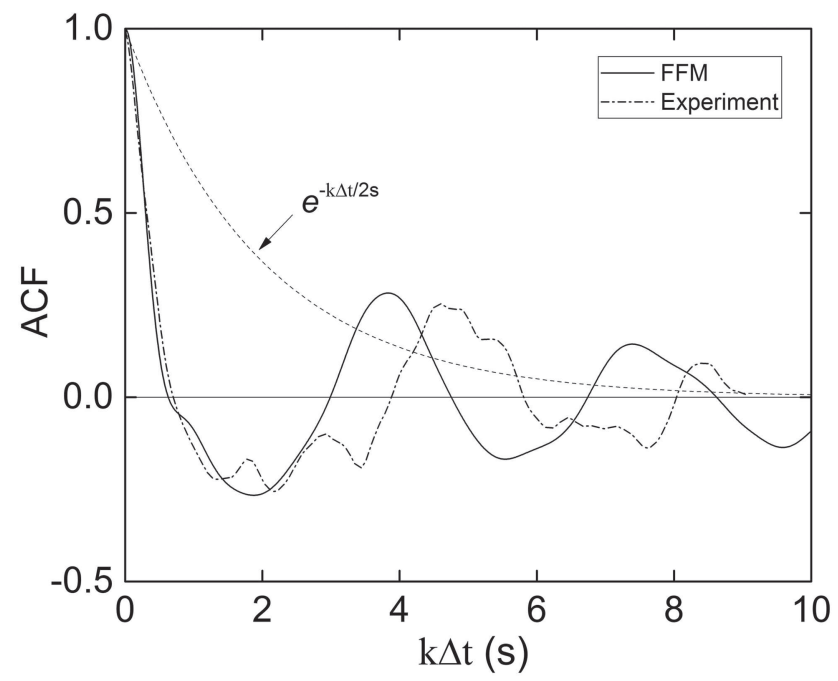

FIG. 7. Comparison of ACF from experiments and numerical simulations. The dashed curve represents the exponential decay based on FTLE. signal from numerical simulations and experiments given by

$$
\operatorname{ACF}(\mathrm{k} \Delta \mathrm{t})=\frac{\mathbf{E}\left[\alpha_{1, j}, \alpha_{1, j+k}\right]}{\sqrt{\operatorname{Var}\left[\alpha_{1, j}\right] \operatorname{Var}\left[\alpha_{1, j+k}\right]}},
$$

where $\operatorname{Var}\left[\alpha_{1, j}\right]$ and $\operatorname{Var}\left[\alpha_{1, j+k}\right]$ represent the variance of liquid volume fraction at two instants having a lag $k \Delta t$. Chaotic systems exhibit an exponential decay of the ACF characterized by a time scale related to the loss of predictability. Figure 7 shows the ACF measurements from the experiments and numerical simulations. While the rate of decay of ACF is not the same as the FTLE, they are related for certain mathematical functions. ${ }^{28,29}$ More specifically, the rate of decay of correlation is bounded by the Largest Lyapunov Exponent in such cases. The exponential curve (indicated by a dashed line) in the figure represents this bound and is plotted using FTLE calculated at $2.4^{\circ}$. The relation ${ }^{28,29}$ holds true for this case of the wavy-stratified flow as well.

\section{CONCLUSIONS}

This study highlights the ability of a 1-D FFM to capture the chaotic behavior of the interface in a wavy-stratified fluid-fluid flow. FFM simulations have been used to extend the stability analysis past the linear theory. The numerical implementation of the hyperbolicdispersive FFM is verified using a spectral convergence test, which is not commonly done for multiphase flows. Qualitatively similar dynamics are reproduced relative to a finite length and time experiment. Quantitative comparison is limited due to differences between the experimental and simulated systems. However, averaged statistical measures are compared including the dominant wavelength and, for the first time, the interfacial ACF. Numerical analysis has revealed at least three oft-cited markers for identifying chaotic systems: (i) infinitesimally close states diverge exponentially quantified by positive Lyapunov exponent(s), i.e., sensitive dependence on the initial condition, (ii) the ACF decays exponentially and, further, is of the same order of magnitude as the FTLE, and (iii) a continuous wave amplitude spectrum in the Fourier space. It should also be noted that the divergence of nearby states saturates, i.e., trajectories are bounded by an attractor (likely strange). The results substantiate a strong relationship between chaos in the fluid-fluid system and the degree of the KHI. Finally, although quantitative comparisons with the experimental data were limited, the decay rate of the ACF is in a fairly good agreement.

\section{NOMENCLATURE}

A Area

$D \quad$ Diameter of the pipe

$L \quad$ Length of the pipe

$g_{x} \quad$ Gravitational Constant

$H \quad$ Height of the channel

$h$ Height of the interface

$k_{\lambda}$ Wave number

$P_{i} \quad$ Interfacial perimeter

$P_{w i} \quad$ Wetted perimeter

$r_{\rho} \quad$ Density ratio

$u_{i} \quad$ Velocity of $i$ th phase 


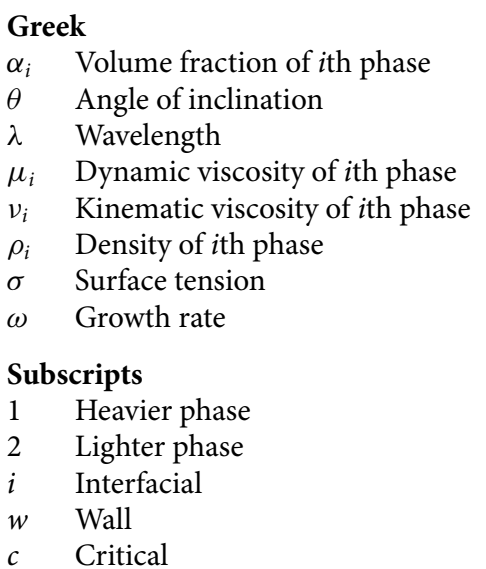

\section{REFERENCES}

'O. E. Lanford, "The strange attractor theory of turbulence," Annu. Rev. Fluid. Mech. 14, 347-364 (1982).

${ }^{2}$ D. V. Pence and D. E. Beasley, "Chaos suppression in gas-solid fluidization," Chaos 8, 514-519 (1998).

${ }^{3}$ W. D. Fullmer and C. M. Hrenya, "Are continuum predictions of clustering chaotic?," Chaos 27, 031101 (2017).

${ }^{4}$ R. J. Wilson, P. A. Delamere, F. Bagenal, and A. Masters, "Kelvin-Helmholtz instability at Saturn's magnetopause: Cassini ion data analysis," J. Geophys. Res., [Space Phys.] 117, A03212, https://doi.org/10.1029/2011JA016723 (2012).

${ }^{5}$ T. Al-Wahaibi and P. Angeli, "Transition between stratified and non-stratified horizontal oil-water flows. Part I: Stability analysis,” Chem. Eng. Sci. 62, 2915-2928 (2007).

${ }^{6} \mathrm{M}$. Duponcheel, S. Mimouni, S. Fleau, and Y. Bartosiewicz, "Experimental and numerical investigations of a two-phase wavy flow," Nucl. Eng. Des. 321, 199-218 (2017).

${ }^{7} \mathrm{~J}$. A. Thorpe, "Experiments on the instability of stratified shear flow: Immiscible fluids," J. Fluid Mech. 39, 25-48 (1969).

${ }^{8}$ A. Vaidheeswaran, W. D. Fullmer, K. Chetty, R. G. Marino, and M. L. de Bertodano, "Stability analysis of chaotic wavy stratified fluid-fluid flow with the $1 \mathrm{D}$ fixed-flux two-fluid model," in Proceedings of ASME 2016 HT/FEDSM/ICNMM (American Society of Mechanical Engineers, 2016).

${ }^{9}$ J. D. Ramshaw and J. A. Trapp, "Characteristics, stability and short wavelength phenomena in two-phase flow equation systems," Nucl. Sci. Eng. 66, 93-102 (1978).

${ }^{10}$ D. Barnea and D. Taitel, "Non-linear interfacial instability of separated flow," Chem. Eng. Sci. 49, 2341-2349 (1994).

${ }^{11}$ Y. Taitel and A. E. Dukler, "A model for predicting flow regime transitions in horizontal and near horizontal gas-liquid flow,” AIChE J. 22, 47-55 (1976).
${ }^{12}$ M. L. de Bertodano, W. Fullmer, and A. Vaidheeswaran, "One-dimensional two-equation two-fluid model stability," Multiphase Sci. Technol. 25, 133-167 (2013).

${ }^{13}$ D. Picchi, S. Correra, and P. Poesio, "Flow pattern transition, pressure gradient, hold-up predictions in gas/non-Newtonian power-law fluid stratified flow," Int. J. Multiphase Flow 63, 105-115 (1994).

${ }^{14}$ W. D. Fullmer, "Dynamic simulation of wavy-stratified two-phase flow with the one-dimensional two-fluid model," Ph.D. dissertation (Purdue University, 2014).

${ }^{15} \mathrm{R}$. Krishnamurthy and V. H. Ransom, "A nonlinear stability study of the relap $5 / \bmod 3$ two-phase model," in Japan-US Seminar on Two-phase Flow Dynamics (Berkeley, CA, 1992), pp. 51-66.

${ }^{16} \mathrm{M}$. L. de Bertodano, W. Fullmer, A. Clausse, and V. Ransom, Two-Fluid Model Stability, Simulation and Chaos (Springer International Publishing, 2017).

${ }^{17}$ J. Canny, "A computational approach to edge detection," IEEE Trans. Pattern Anal. Mach. Intell. PAMI-8, 679-698 (1986).

${ }^{18} \mathrm{M}$. Ishii and T. Hibiki, Thermo-fluid Dynamics of Two-Phase Flow (Springer, New York, NY, 2011).

${ }^{19}$ D. A. Drew and S. L. Passman, Theory of Multicomponent Fluids (Springer, New York, NY, 1999).

${ }^{20} \mathrm{C}$. Morel, Mathematical Modeling of Disperse Two-Phase Flows (Springer International Publishing, 2015)

${ }^{21}$ A. Vaidheeswaran and M. L. Bertodano, "Stability and convergence of computational eulerian two-fluid model for a bubble plume," Chem. Eng. Sci. 160, 210-226 (2017).

${ }^{22} \mathrm{~N}$. Andritsos and T. Hanratty, "Interfacial instabilities for horizontal gas-liquid flows in pipelines," Int. J. Multiphase Flow 13, 583-603 (1987).

${ }^{23} \mathrm{M}$. Arai, "Characteristics and stability analyses for two-phase flow equation systems with viscous terms," Nucl. Sci. Eng. 74, 77-83 (1980).

${ }^{24}$ W. D. Fullmer, M. A. L. de Bertodano, M. Chen, and A. Clausse, "Analysis of stability, verification and chaos with the Kreiss-Yström equations," Appl. Math. Comput. 248, 28-46 (2014).

${ }^{25} \mathrm{P}$. H. Gaskell and A. K. C. Lau, "Curvature-compensated convective transport: Smart, a new boundedness-preserving transport algorithm," Int. J. Numer, Methods Fluids 8, 617-641 (1988).

${ }^{26}$ S. Gottlieb and C.-W. Shu, "Total variation diminishing runge-kutta schemes," Math. Comput. 67, 73-85 (1998).

${ }^{27}$ W. D. Fullmer, A. Clausse, A. Vaidheeswaran, and M. A. L. de Bertodano, "Numerical solution of wavy-stratified fluid-fluid flow with the one-dimensional two-fluid model: Stability, boundedness, convergence and chaos," in ASME 2014 4th Joint US-European Fluids Engineering Division Summer Meeting Collocated with the ASME 2014 12th International Conference on Nanochannels, Microchannels, and Minichannels (American Society of Mechanical Engineers, 2014), pp. V002T20A006-V002T20A006.

${ }^{28}$ R. Badii, K. Heinzelmann, P. F. Meier, and A. Politi, "Correlation functions and generalized Lyapunov exponents,” Phys. Rev. A 37, 1323-1328 (1988).

${ }^{29}$ J. Slipantschuk, O. F. Bandtlow, and W. Just, "On the relation between Lyapunov exponents and exponential decay of correlations," J. Phys. A Math. Theor. 46, 075101 (2013). 